\title{
Managing nonspecific abdominal pain in children and young people
}

\author{
Neil Chanchlani MBChB MSc, Thomas D. Walters MBBS MSc, Richard K. Russell MBChB PhD
}

Cite as: CMAJ 2020 November 30;192:E1639-40. doi: 10.1503/cmaj.202555

See the related research at www.cmaj.ca/lookup/doi/10.1503/cmaj.201055

I $\mathrm{n}$ related research, Poonai and colleagues performed a randomized, blinded, double-dummy, single-centre trial to compare the efficacy of hyoscine butylbromide and acetaminophen in children and young people who presented to the emergency department with nonspecific, colicky abdominal pain. ${ }^{1}$ Nonspecific abdominal pain is common among children presenting to both primary and emergency care. In the absence of clear alarm features, other systemic features, objective evidence of poor growth or positive family history of a gastrointestinal disorder (e.g., inflammatory bowel disease, celiac disease, peptic ulcer disease), most patients can be managed safely in primary care without further bloodwork or imaging. Parents are usually advised to monitor symptoms and offer simple analgesia, such as acetaminophen and ibuprofen. Only $5 \%$ of children presenting to primary care will require referral to secondary care. ${ }^{2}$ However, establishing a positive relationship between the clinician and the child and their parent is important from the first presentation to lay the foundation for a potential future therapeutic relationship and complex interventions.

In their large trial, Poonai and colleagues observed a clinically significant reduction in pain scores among two-thirds of participants, all of whom received analgesia safely; however, hyoscine butylbromide was not found to be superior to acetaminophen. ${ }^{1}$ Although simple analgesia is known to be well-tolerated and safe in children, evidence regarding its efficacy in children with undifferentiated or chronic abdominal pain is lacking, ${ }^{3,4}$ so the related research adds usefully to the literature. However, 2 major study limitations warrant further consideration.

First, the trial enrolled only children aged 8-17 years who were screened between the hours of 1700 and 2300 , thereby biasing participant selection by excluding preschool-age and early-school-age children and likely those whose pain developed during the school day. Children as young as 4 years commonly present with nonspecific abdominal pain. Younger children may have been excluded from the study owing to drug formulation or parental acceptability concerns. However, younger children's pain is poorly understood, often overlooked by caregivers and

\section{KEY POINTS}

- Children present frequently to primary and emergency care with nonspecific abdominal pain.

- Only a few treatment options exist to manage colicky, undifferentiated pain; however, most children will not present more than once or twice to health services for this problem.

- Undifferentiated abdominal pain is poorly understood but likely multifactorial, arising from a combination of sensitizing medical events, psychosocial events and visceral hyperalgesia.

- Among children who seek health care for this problem, $20 \%-25 \%$ go on to be diagnosed with a disorder of the brain-gut axis, "a functional gastrointestinal disorder," for which international guidelines exist to guide management.

- At first consultation, it is important to establish a positive therapeutic relationship between the clinician and the child and their parents, focusing on optimizing symptom control over unnecessary investigation and medicines.

possibly less responsive to analgesia, so the inclusion of younger children would have provided much-needed information. Abdominal pain that develops during the school day is more likely to have a functional cause, for which medication is less likely to be beneficial. ${ }^{5}$

Second, improvement in abdominal pain is multifactorial and usually not attributable to medical therapy alone. ${ }^{6,7}$ The ethics committee granting approval for the related research did not allow a placebo arm in the trial. However, the findings of several trials studying interventions for functional abdominal pain have suggested that a strong placebo effect may influence failure to show a significant effect of an intervention. ${ }^{8}$

Nevertheless, the findings of the related study are reassuring, as hyoscine butylbromide is already approved for use in children aged 6 years or older for smooth muscle spasm of the gastrointestinal and genitourinary tracts, including irritable bowel syndrome and bowel colic. Poonai and colleagues' findings are novel for the pediatric population and consistent with those of a randomized controlled trial conducted among adults attending 
the emergency department, where acetaminophen was found to be as effective as hyoscine butylbromide, or a combination of the 2 , in treating undifferentiated abdominal pain. ${ }^{9}$

Determining response to any analgesic requires reassessment of pain, but objective markers of improvement, such as whether the child is easily distracted and is able to eat, drink, or pass urine and stool, can be assessed during an observation period in hospital or in a follow-up telephone call to determine the next course of action. Parents' views and assessment of progress should always be taken into account.

For children referred on to secondary care for further investigation of abdominal pain, there are few options other than simple analgesia for pain management. Since 2013, codeine is no longer approved for use in children younger than 12 years, and opioid-containing medicines can paradoxically worsen pain if it is related to constipation, as is much abdominal pain. Children who have already received simple analgesia may be offered an anticholinergic analgesic, such as hyoscine butylbromide, as a next step in the face of persistent pain.

Most children will present to health services only once or twice for nonspecific abdominal pain. However, 20\%-25\% will go on to be diagnosed with a disorder of the brain-gut axis, alternatively termed "functional gastrointestinal disorder," 10 such as functional dyspepsia, abdominal migraine, irritable bowel syndrome and functional abdominal pain not otherwise specified. The 2016 Rome IV criteria provide the latest diagnostic, investigative and management guidance for such disorders. ${ }^{11}$

While the pathophysiology underlying acute undifferentiated or chronic abdominal pain in children is incompletely understood, the pain likely arises from a combination of sensitizing medical events (e.g., bowel distention, inflammation or dysmotility), sensitizing psychosocial events (e.g., family stress, anxiety or a particular psychological coping style) and early-life events that lead to visceral hyperalgesia. ${ }^{11}$ Diagnosing a functional gastrointestinal disorder will usually take time - often months - during which patients are likely to experience a diminished quality of life. ${ }^{12,13}$ If symptoms are disruptive to the child's school attendance or hobbies, use of analgesia may be beneficial, particularly if early use breaks a pain cycle, thus reducing the consequences of pain for the child. However, this potential benefit should be balanced against the possibility that habitual or reflexive medication use could become an unnecessary part of the solution.

The establishment of a positive therapeutic relationship between the clinician and the child and their parent is equally important to any medication use. Clear and careful explanation of brain-gut interactions by clinicians who see the child at initial presentation; reinforcement that investigations and medicines are usually unnecessary; and discussion of behavioural management options with a focus on optimising symptom coping skills, ${ }^{7,11}$ will build a foundation for this positive therapeutic relationship.

\section{References}

1. Poonai N, Kumar K, Coriolano K, et al. Hyoscine butylbromide versus acetaminophen for nonspecific colicky abdominal pain in children: a randomized controlled trial. CMAJ 2020;192:E1612-9.

2. Gieteling MJ, Lisman-van Leeuwen Y, van der Wouden JC, et al. Childhood nonspecific abdominal pain in family practice: incidence, associated factors, and management. Ann Fam Med 2011;9:337-43.

3. Cooper TE, Fisher E, Anderson B, et al. Paracetamol (acetaminophen) for chronic non-cancer pain in children and adolescents. Cochrane Database Syst Rev 2017;8:CD012539.

4. Kanabar DJ. A clinical and safety review of paracetamol and ibuprofen in children. Inflammopharmacology 2017;25:1-9.

5. Assa A, Ish-Tov A, Rinawi F, et al. School attendance in children with functional abdominal pain and inflammatory bowel diseases. J Pediatr Gastroenterol Nutr 2015;61:553-7.

6. Brusaferro A, Farinelli E, Zenzeri L, et al. The management of paediatric functional abdominal pain disorders: latest evidence. Paediatr Drugs 2018;20:235-47.

7. Chiou E, Nurko S. Management of functional abdominal pain and irritable bowel syndrome in children and adolescents. Expert Rev Gastroenterol Hepatol 2010;4:293-304.

8. Hoekman DR, Zeevenhooven J, van Etten-Jamaludin FS, et al. The placebo response in pediatric abdominal pain-related functional gastrointestinal disorders: a systematic review and meta-analysis. J Pediatr 2017;182:155-63.e7.

9. Remington-Hobbs J, Petts G, Harris T. Emergency department management of undifferentiated abdominal pain with hyoscine butylbromide and paracetamol: a randomised control trial. Emerg Med J 2012;29:989-94.

10. Scarpato E, Kolacek S, Jojkic-Pavkov D, et al.; MEAP Group. Prevalence of functional gastrointestinal disorders in children and adolescents in the Mediterranean region of Europe. Clin Gastroenterol Hepatol 2018;16:870-6.

11. Hyams JS, Di Lorenzo C, Saps M, et al. Childhood functional gastrointestinal disorders: child/adolescent. Gastroenterology 2016;150:1456-68.e2.

12. Robin SG, Keller C, Zwiener R, et al. Prevalence of pediatric functional gastrointestinal disorders utilizing the Rome IV criteria. J Pediatr 2018;195:134-9.

13. Varni JW, Bendo CB, Nurko S, et al.; Pediatric Quality of Life Inventory (PedsQL) Gastrointestinal Symptoms Module Testing Study Consortium. Health-related quality of life in pediatric patients with functional and organic gastrointestinal diseases. J Pediatr 2015;166:85-90.

Competing interests: Neil Chanchlani is funded by a research fellowship from Crohn's and Colitis UK. Thomas Walters receives research support from Janssen Canada, Merck Canada and AbbVie Canada, and has consulted for Janssen Canada, Merck Canada, AbbVie Canada and Ferring. Richard Russell is supported by an NHS Research Scotland Senior Research Fellowship and has received speaker fees, travel support, and/or participated in medical board meetings with Nestlé, AbbVie, Dr. Falk Pharma, Takeda, Napp, Mead Johnson, Nutricia, and 4D Pharma.

This article was solicited and has not been peer reviewed.

Affiliations: Department of Paediatrics (Chanchlani), Royal Devon and Exeter Hospital, Exeter, UK; Department of Paediatric Gastroenterology (Walters), The Hospital for Sick Children, Toronto, Ont.; Department of Paediatric Gastroenterology (Russell), Royal Hospital for Sick Children, Edinburgh, UK

Contributors: Neil Chanchlani and Richard Russell conceived the idea and drafted the manuscript. Thomas Walters provided critical advice and recommendations for revision. All authors approved the final version for publication and agree to be accountable for all aspects of the work.

Disclaimer: Neil Chanchlani is an associate editor for CMAJ and was not involved in the editorial decision-making process for this article.

Correspondence to: Neil Chanchlani, nchanchlani@doctors.org.uk 